\title{
FROM A METAL VAPOR LASER PROJECTION MICROSCOPE TO A LASER MONITOR (BY THE 50-YEAR ANNIVERSARY OF METAL VAPOR LASERS)
}

\author{
Evtushenko G.S. \\ National Research Tomsk Polytechnic University, \\ 30 Lenin Ave., Tomsk, 634050 Russia
}

\begin{abstract}
The paper presents the history of active optical systems development from a laser projection microscope to a laser monitor. The examples of object visualization and diagnostics of high speed processes hidden by the intense background radiation are discussed. These are the processes of laser-surface interaction, self-propagating high-temperature synthesis (SHS), the corona discharge in the air, the nanoparticle production process using a high-power fiber laser, and etc. The results obtained by different research groups suggest that high-speed metal vapor brightness amplifiers and active optical systems based on them need further research, development and novel applications.
\end{abstract}

Keywords: active optical system, metal vapor laser, microscope, monitor, visualization, background radiation, high speed amplifier, object, processing

\section{INTRODUCTION}

Methods of visual and optical monitoring with the use of active optical systems are rather popular in non-destructive testing and diagnostics of fast processes. In the 1970-s one of the first and most efficient active optical systems was a laser projection microscope. The key element of this microscope was a brightness amplifier [1]. The first brightness amplifiers, which were based on solid state active media and gas media on the transitions in the inert gases, were designed in the 1960-s [2-5]. The metal vapor active medium, copper in particular, was first used as a brightness amplifier in 1970 [6]. Copper vapor lasers and brightness amplifiers based on their active media became more popular after the implementation of the self-heating mode [7]. This allowed to obtain high metal vapor laser performance and to design a laser projection microscope, and a laser monitor afterwards, for the visualization of objects and fast processes, including those blocked from viewing by the background radiation.

\section{REQUIREMENTS FOR THE BRIGHTNESS AMPLIFIERS OF ACTIVE OPTICAL SYSTEMS}

In $[8,9]$ the basic requirements for active media used as brightness amplifiers were formulated. Taking into account the contemporary data they can be represented as follows.

The medium of the amplifier must be optically homogeneous to lower the distortions in the transmitted information; High amplification in a narrow spectral range which will allow the instrument to operate in an ASE mode to illuminate the object and perform narrowband filtration of the reflected signal when it is amplified;

Geometric dimensions and angular aperture must ensure the passing of the light beams without optical losses; The power (energy) of radiation at the output of the brightness amplifier must be sufficient to be used not only in experimental but also practical applications (visual monitoring);

The active medium must operate in the continuous or pulsed-periodic mode, which allows to obtain image of an object in real time mode.

The most common media satisfying the following conditions are atomic gas media on self-terminating transitions in metal vapors due to their homogeneity, a possibility of obtaining a high degree of monochromaticity (high spectral brightness) of radiation, and pulsed-periodic operating mode. 


\section{LASER PROJECTION MICROSCOPE AND LASER MONITOR: OPTICAL SCHEMES AND APPLICATIONS}

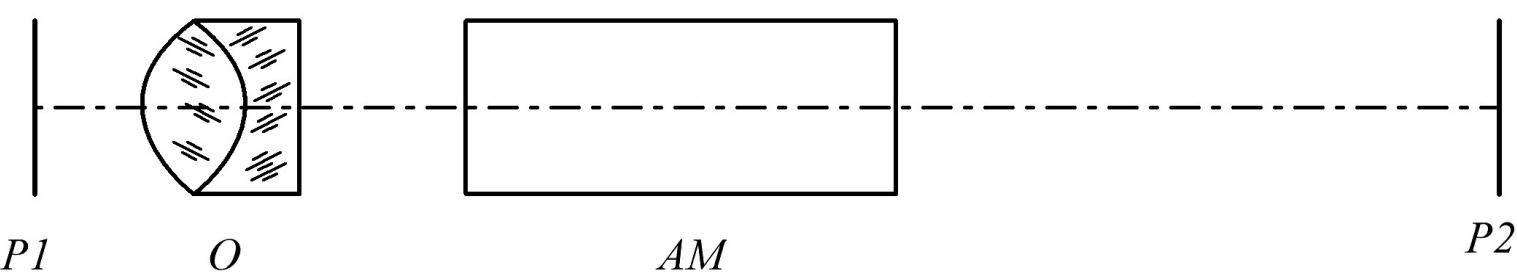

Fig.1 Simplified scheme of a laser projection microscope [1].

Fig. 1 shows the so-called monostatic scheme of a microscope. The object placed in P1 plane is illuminated by the ASE of the active medium. Then the signal reflected from the object passing back through the active medium is amplified and produces an image amplified by the active optical system on the screen, which is placed in P2 plane [1]. It is evident that in such a configuration the distance between the active medium and the object is limited by the time of inversion in the active medium. This typical time of inversion for MVLs on self-terminating transitions is $20-30$ ns. When the distance between the object and the active medium does not exceed $1 \mathrm{~m}$, the limitation mentioned above becomes insignificant, which allows to visualize objects (including micro-objects) with the spatial resolution comparable to the wavelength ( 0.5 $\mu \mathrm{m})$. Fig. 2 shows an example of visualization of a piece of mica and a slice of onion from [9]. In order to enhance the contrast, the images are obtained by the 'shadow method' when a mirror is placed behind the object with a low reflectance coefficient.

Fig. 2 Amplified images obtained using a 'shadow method' a) piece of a muscovite mica, b) slice of the onion.
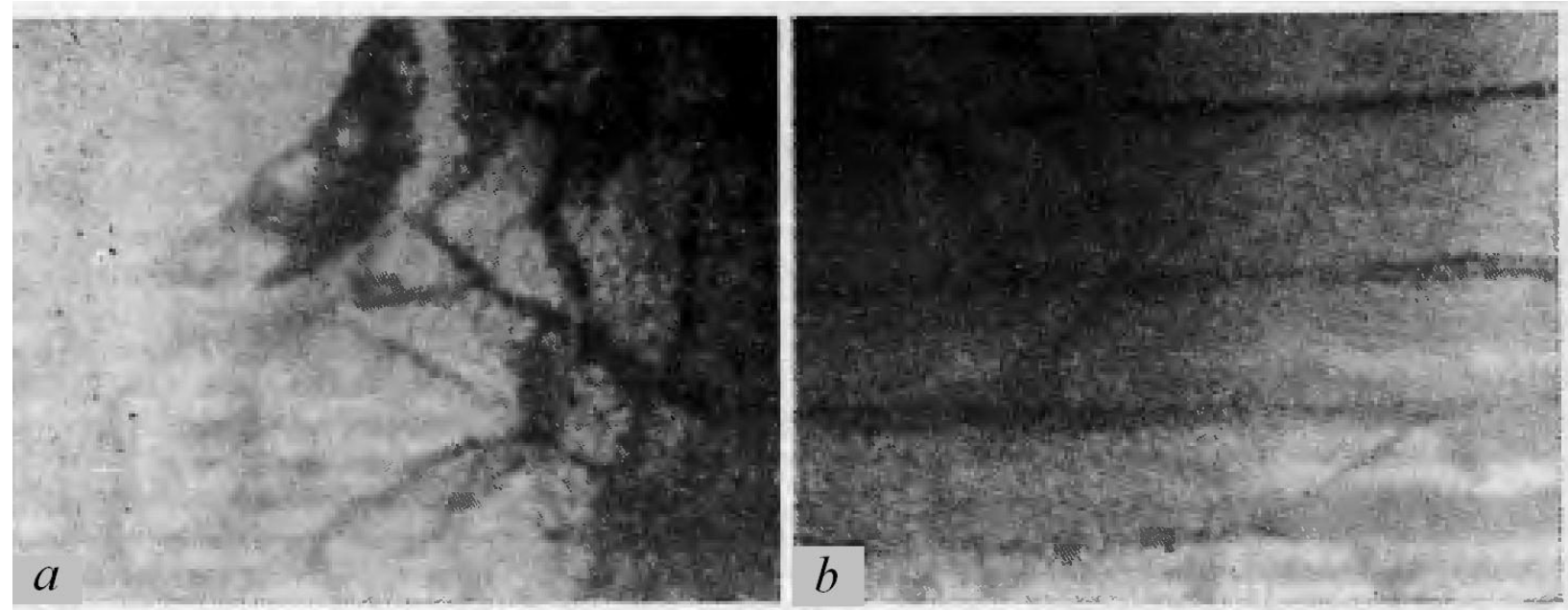

The idea of obtaining an image of an object using a single pulse to take an advantage of pulsed-periodic mode of MVL radiation was offered by prof. G. Petrash (in private discussions). The first to implement this principle in practice were V. Savransky and his colleagues. They used high-speed optical and mechanical image scanning in real-time mode [10]. Fig. 3 shows the results of visualization of bio-objects: infusoria (3a) and the neuron Helix pomatia (3b) registered with the time interval of $800 \mu \mathrm{s}$. The exposition time is $10 \mathrm{~ns}$. The arrows indicate the changes in structural morphology. Unfortunately, the authors could observe the image obtained only from three pulses. It implies that at a PRF of $11 \mathrm{kHz}$ the time resolution could have been $10-4 \mathrm{sec}$. 


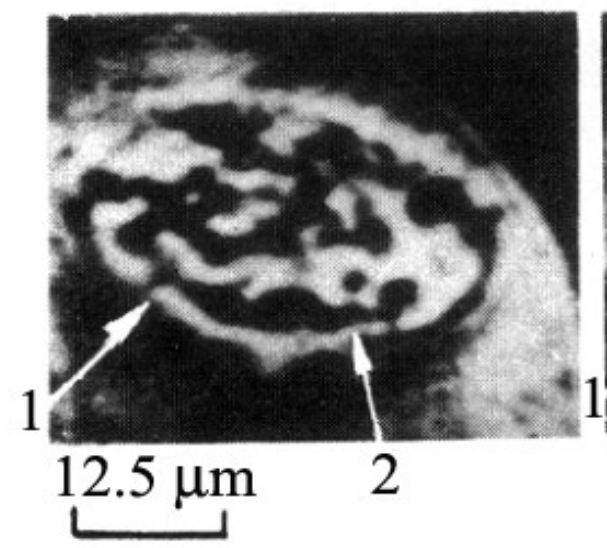

a)

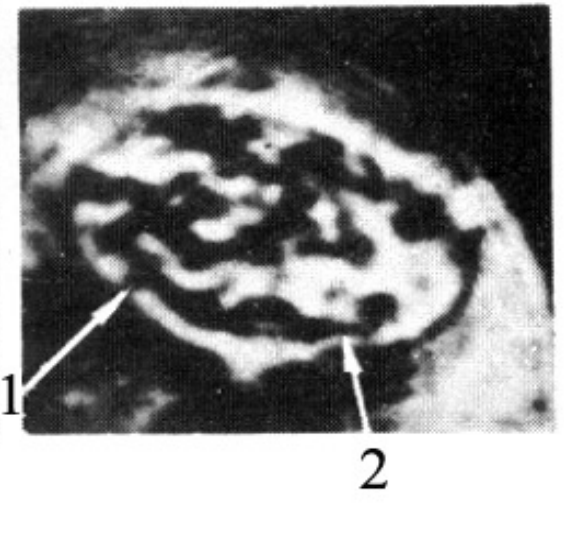

b)

Fig. 3. Images of infusoria (a) and neuron Helix pomatia (b) [12].

In the 1970-1980s of the previous century Russia became a leader in research and development of brightness amplifiers based on metal vapors, and also their application (as an integral part of the laser projection microscope) in science and visual and optical monitoring of integral microchips manufacturing [8-12]. The growth of new technologies related to the impact of energy fluxes on the object (the modification of materials surface, nanostructure materials as well, welding and cutting processes, production of new materials using SHS synthesis, etc.) renewed the interest in active optical systems. These processes are usually blocked from viewing by the background radiation which does not allow to visualize the object or control the process. The influence of the background light can be reduced using the pulsed laser illumination method [13]. However, this method does not allow to completely block the background radiation. Laser active media on self-terminating transitions in metal vapors used as brightness amplifiers are capable of visualizing such objects or processes due to the high spectral brightness and the high gain in the narrow spectral range, and in different spectral regions as well. Pulsed-periodic radiation mode, as it has been previously mentioned, allows to monitor objects and processes with the time resolution corresponding to the pulse repetition frequency (PRF).

It is not yet clear who was the first to suggest to observe an object obscured by the background light, or in other words, to 'look through the flame'. Probably these were I. Klimovsky [14] and G. Abrosimov [15] with their colleagues. It was I. Kilmovsky who introduced the notion of 'laser monitor'. Before that the main terms had been 'laser projection microscope' and 'active optical system with a brightness amplifier' $[9,12]$. O.Buzhinsky with his colleagues proposed a bistatic scheme of a laser monitor when they studied non-destructive testing of the surface of the internal wall of the nuclear reactor. The authors called this instrument for visualization a 'videoscope' $[16,17]$. But in practice this instrument has never been constructed.

An example of laser monitor operation is shown in Fig. 4 [14]. This figure presents the visualization results of the cathode - $\mathrm{K}$ and the anode - $\mathrm{A}$ in the process of the dc current arc combustion. Images were registered using a camera without time resolution, in the presence of the intense background glare of the arc (continuum with the brightness temperature of the order of $5000 \mathrm{~K}$ ). In Fig. $4 \mathrm{~b}$ we can clearly see cathode and anode spots, which are impossible to observe with an ordinary microscope. Similar results are also obtained in [15], but the quality of the images is much lower. 
A)
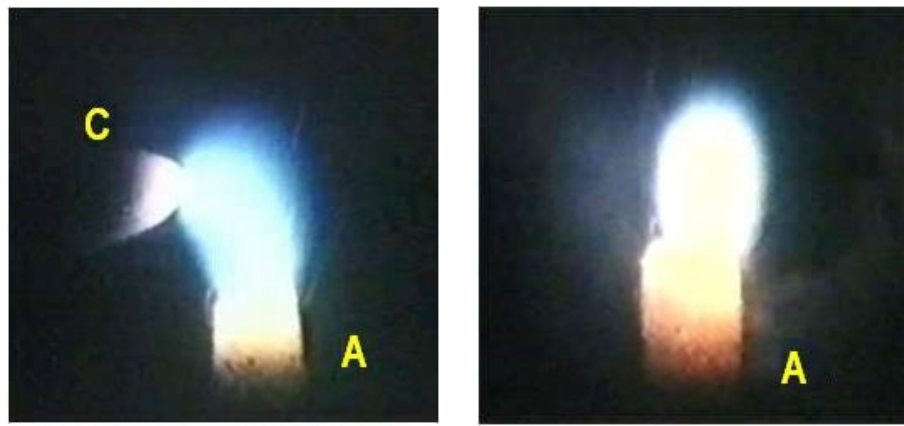

B)
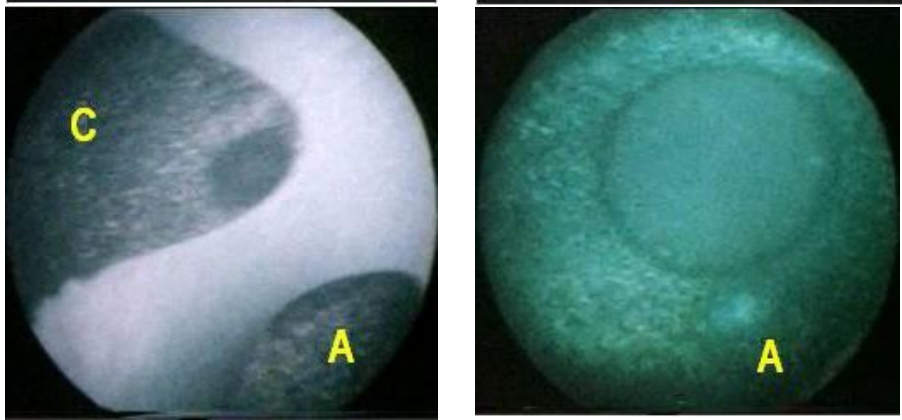

Fig. 4. Images of the carbon arc obtained with a conventional (a) and laser projection (b) microscopes [14].

It is necessary to mention several research groups who have have been studying metal vapor brightness amplifiers (laser monitors). The group of professor Arakelyan have been investigating the visualization of processes of laser-surface interaction. Fig. 5 shows the results of melting of carbon heated by the focused laser radiation in air [18]. The graphite specimen was heated by the focused radiation of YAG:Nd-laser (with the PRF of $150 \mathrm{~Hz}$, the radiation wavelength of $1.06 \mu \mathrm{m}$, the pulse duration of $3 \mathrm{~ms}$ ). The average radiation power was varied from 10 to $80 \mathrm{~W}$. The process of interaction of laser radiation with the graphite surface was observed using a brightness amplifier based on a copper vapor laser.
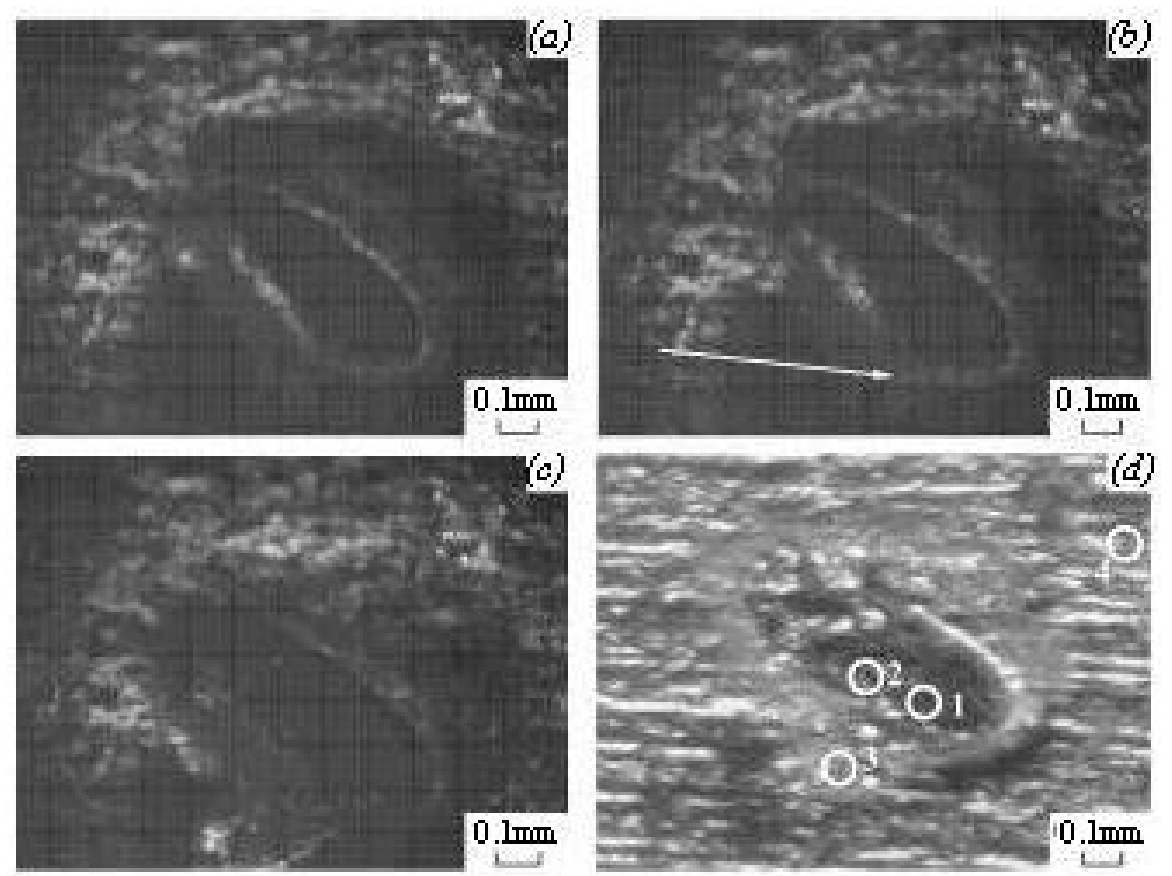

Fig. 5. Images of the evolution of the graphite surface under exposure to laser radiation $(a, b, c)$ and after the end of the experiment (d): (a) the time of interaction $t=184 \mathrm{~ms}$; (b) $t=258 \mathrm{~ms}$; (c) $t=534 \mathrm{~ms}$. Small circles indicate the zones where Raman scattering spectra were obtained. 
Fig. 5 shows three (a), (b), (c) video frames illustrating the surface of the graphite structure in real-time mode when the sample is exposed to the laser radiation. Image (d) is obtained after the end of the exposure with an ordinary optical microscope. It is seen that during the time period under exposure to radiation there appears an oblong cavity on the graphite sample surface. Its size gradually increases and a circle along the border of the crater protruding above the surface is formed. Then in the head part of the crater formed around the region of radiation focus of YAG:Nd-laser the liquid carbon flows out. This results in the destruction of the corresponding part of the circle (the place of destruction is indicated with an arrow) and the liquid carbon flows across the sample surface at a distance of about $0.2 \mathrm{~mm}$ from the crater circle. The time of the liquid carbon leakage outside the cavity does not exceed $0.3 \mathrm{~s}$. Subsequently the motion of melting carbon across the surface stops and it crystallizes. Thus, the use of a laser monitor allows to study the hydrodynamic instabilities and waves induced by pulsed-periodic radiation on the surface of a substance. The results obtained by this research group are presented in detail in [19].

The research group of MEPHI (A. Kuznetzov and others) use a copper vapor laser for the visualization of surface treatment processes, drilling, welding by a fiber laser which are also obscured by the intense broadband glare. The laser monitor allowed to visualize the process of welding at different stages. As it is seen from Fig. 6, the instrument makes it possible to monitor the welding process: formation of small molten pools, wave patterns on the weld seam, flows and fluctuations in the melt [20].
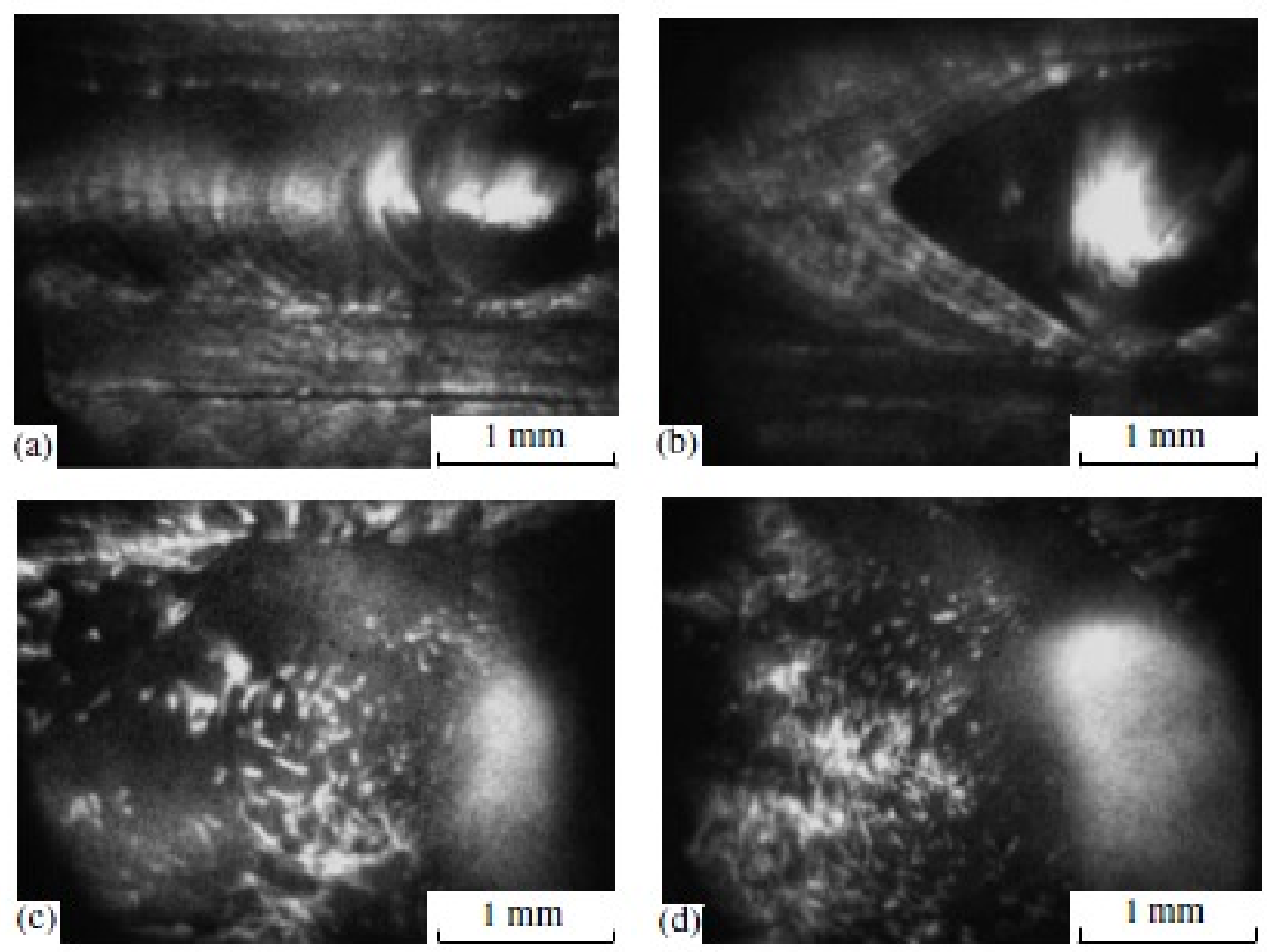

Fig. 6. Image of the weld seam exposed to radiation of a fiber laser operating at a different power: $\mathrm{a}-0.5, \mathrm{~b}-1.0, \mathrm{c}-2.0, \mathrm{~d}-4.0 \mathrm{~kW}$.

The same group obtained important results on the modification of the cathode surface, including cases with the intense background radiation of non-equilibrium low-temperature plasma of erosive capillary discharge [21].

Another study of great interest was carried out by P. Bokhan with his colleagues. They used the UV-radiation of Aulaser $(312.2 \mathrm{~nm})$ for the visualization of semiconductor units using a laser projection microscope with a micron spatial resolution [22].

It is important to note that by 2000 the brightness amplifiers on transitions of different metals $(\mathrm{Cu}, \mathrm{Au}, \mathrm{Ba}, \mathrm{Mn}, \mathrm{Pb}, \mathrm{Sr})$ and their compounds covered a wide spectral range from 0.3 to $1.5 \mu \mathrm{m}[6,22-26]$. In cases when the amplification of the active medium was not high enough (in particular, on recombination transitions of strontium atom - less than $20 \mathrm{~dB} / \mathrm{m}$ ) 
the authors used multi-pass schemes to design a projection microscope. At that time there were almost no studies on single pass imaging (except for [10]).

After the first presentation of our results on designing and testing of the device for visualization [27] in 2009 we started to refer to the device capable of visualizing objects and fast processes blocked by the background radiation in real-time mode as a 'laser monitor'. Typical PRF of metal vapor lasers are $5-15 \mathrm{kHz}$, whereas the PRF of metal halide vapor lasers is a bit higher $(15-30 \mathrm{kHz})[7,12]$. Therefore, the brightness amplifier in our work is based on copper bromide vapor active medium. At present, the hardware-software system referred to as 'laser monitor' consists of a copper bromide vapor brightness amplifier, optical scheme, high-speed digital camera, frame-by-frame imaging system, software for image processing [28]. This system allows to obtain the time resolution of 10-4 - 10-5 sec and spatial resolution of 1 $\mu \mathrm{m}$. The instrument has been tested on test and real objects. Fig. 7 shows the visualization of SHS process. The experiments were made with researchers from the Department of structural macrockinetics of Tomsk scientific centre SB RAS . The field of view was $5 \times 5 \mathrm{~mm}$. In the first experiments images were registered with the frame rate of $420 \mathrm{fps}$, without frame-by-frame imaging. The brightness temperature of the SHS process was $1500-4500 \mathrm{~K}$. It is obvious that it is impossible to study structural transformations using conventional methods of optical diagnostics because of the intense background glare that obscures the process.

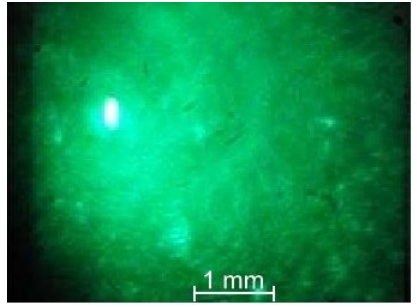

$0 \mathrm{~ms}$

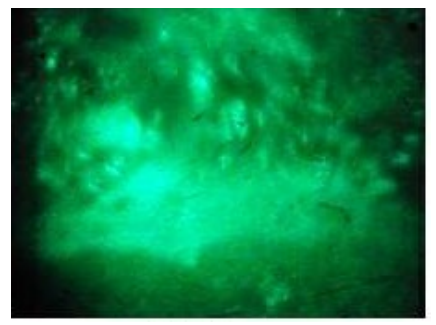

$210 \mathrm{~ms}$

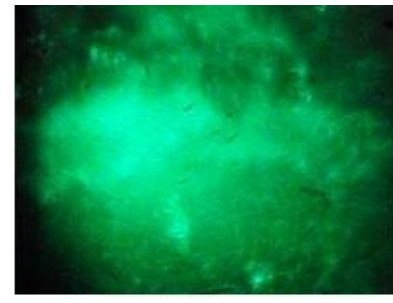

$155 \mathrm{~ms}$

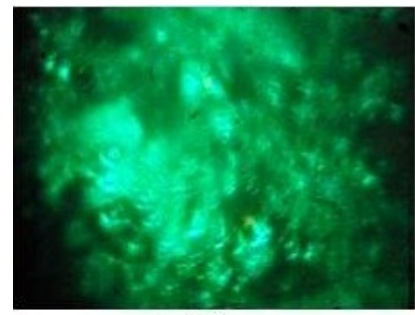

$300 \mathrm{~ms}$

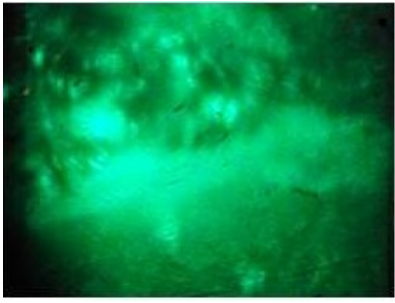

$180 \mathrm{~ms}$

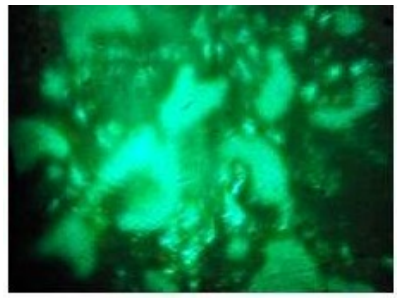

$4500 \mathrm{~ms}$

Fig. 7. Images of the combustion process of $\mathrm{FeTiO} 3+\mathrm{SiO} 2+\mathrm{Si}+\mathrm{Al}+\mathrm{C}$ structure

Key moments of the process such as the beginning of the combustion $(0 \mathrm{~ms})$, the combustion process itself (155 - 300 $\mathrm{ms})$ with the liquid phase, and crystallization $(4500 \mathrm{~ms})$.

We also used an upgraded laser monitor with frame-by-frame imaging for the visualization of the combustion process of SHS structures [29]. One of these structures was a compound $(65 \% \mathrm{FeTiO} 3+35 \% \mathrm{Al})+35 \% \mathrm{Kaoline}$. The frame rate was $2808 \mathrm{fps}$, the field of view was $1.5 \times 1.5 \mathrm{~mm}$.

Fig. 8 presents the experimental results. Each frame is formed by a single radiation pulse with the duration of 40 ns. As a result, the time relation of the device is higher than in the previous case. The image quality is also better as there arise no distortions in the interpulse period. The combustion profile is uneven. At the initial moment of the combustion the surface material is absorbed (upward movement) and pores are formed. This process is followed by the heating of the other part of the structure and liquid and solid phases. 


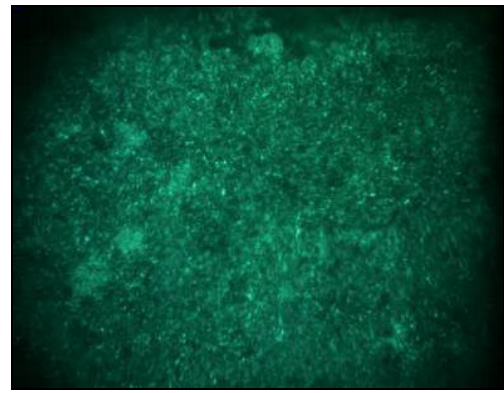

$0 \mathrm{~ms}$

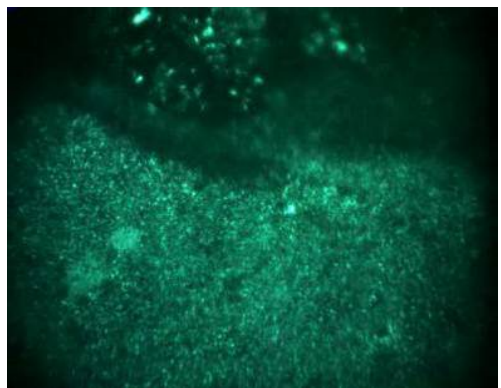

$250 \mathrm{~ms}$

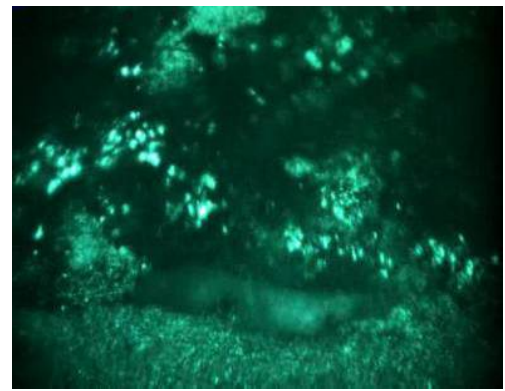

$550 \mathrm{~ms}$

Fig. 8. Frame-by-frame imaging of the combustion process of SHS structure $(65 \% \mathrm{FeTiO} 3+35 \% \mathrm{Al})+35 \% \mathrm{Kaoline}$

The obtained results demonstrate the possibility of studying structural transformations during the SHS process and estimating the speed of this process.

Research groups of V. Tarasenko (IHCE SB RAS) and V.Osipov (EIP UD RAS) visualized the corona discharge in air to study the nanoparticle production process under the radiation of a high-power fiber laser [30, 31]. For these experiments, a small model of a laser monitor was used. In [30] the authors studied the plasma of the corona discharge with a small reflectance coefficient. However, the optical inhomogeneity of the discharge produced by the thermal impact can be visualized using a 'shadow method' of the laser monitor to measure the speed of its distribution. In [31] the use of the laser monitor for visualization allowed to block the intense glare of the laser torch and to study its dynamics in detail.

\section{CONCLUSIONS}

The results obtained by the research groups discussed in this paper suggest that the study of brightness amplifiers and laser monitors based on them is a hot topic nowadays. Primarily we will have to widen the range of applications of the laser monitor taking into account the possibility of imaging through the intense glare. Visual and optical monitoring of remote objects that are difficult to access, or not available, is also of interest nowadays. This will require the enhancement of the high speed equipment and methods of processing. However, it is necessary to solve a number of problems. At present, the maximum PRFs of MVLs reach $1 \mathrm{MHz}[32,33]$. However, as the PRF increases, the pulse duration and the ASE pulse energy decrease. This significantly limits the distance between a brightness amplifier and an object. The aperture of the radiation beam decreases, its profile gets worse, which reduces the field of view. It is also of importance to reduce the size, weight and reliability of the devices, and perform the visualization in different spectral ranges. A possible solution of overcoming the presented challenges is the use of metal vapor active media with modified kinetics, the development of new ways of pumping active media. In particular, the use of the reduced energy input into the discharge [34] and the use of active elements with the inner reactor of metal halides [35] can be mentioned here. All these domains of investigation have a background and advances to be implemented in future.

\section{ACKNOWLEDGMENTS}

The author is grateful to his colleagues V. Sukhanov, D. Shiyanov, F. Gubarev, S. Torgaev, M.Trigub, T. Evtushenko, D. Chertikhina for their help and precious comments.

The paper was supported by Russian Science Foundation, project No 14-19-00175 "High Speed Metal Vapor Brightness Amplifiers".

\section{REFERENCES}

[1] Zemskov, K.I., Isaev, A.A., Kazaryan, M.A., Petrash, G.G., "Laser projection microscope”, Soviet Journal of Quantum Electronics, (1), 14-15 (1974).

[2] Geusic, J.E., Skovil, H.E.D., "A unidirectional traveling wave optical masers", Bell Systems Technology, 5 (41), 1371-1398 (1962).

[3] Lanczi, E.R., "Amplification in a thick ruby lens", Applied Optics, , 5 (2), 255-257 (1965).

[4] Basov, N.G., Grasuyk A.Z., Zubarev I.G., Tevelyev L.V., "Regenerative optical quantum amplifier", Moscow, "Nauka". Trudy FIAN, 31, 74-95 (1965). 
[5] Hardy, W.A., "Active images formation in lasers", IBM Journal of Research and Development, .9 (1), 31-35 (1965)

[6] Rabinovitz, P, Chimenti, R., "Super-radiant illuminator projector”, JOSA, 60, 1577-1778 (1970).

[7] Isaev, A.A., Kazaryan M.A., Petrash G.G., "Effective copper vapor pulsed laser with high average output power", Pis'ma ZHETF, 16 (1), pp. 40-42 (1972).

[8] Zemskov, K. I. , Isaev, A. A. , Kazaryan, M. A. , Petrash, G. G., "Investigation of principal characteristics of a laser projection microscope", Soviet Journal of Quantum Electronics, 3 (1), 35-43 1976.

[9] Petrash, G.G., [Optical Systems with brightness amplifiers] [In Russian], Moscow: Nauka. Trudy FIAN, Vol. 206 (1991).

[10] Morozova, E.A., Prokhorov A.M., Savranskii V.V., Shafeev G.A., "High-speed frame-by-frame registration of images of biological objects with use of a laser projection microscope", Doklady Akademii Nauk (Proceedings of the Russian Academy of Sciences), 261 (6), 1460-1462 (1981).

[11] Zemskov K. I. , Kazaryan M. A., Matveev V. M., Petrash G. G. , Samsonova M. P., Skripnichenko A. S. , "Laser machining of objects with simultaneous visual monitoring in a copper vapor oscillator-amplifier system", Soviet Journal of Quantum Electronics, 11(2), 418-421 (1984).

[12] Pulsed Metal Vapor Lasers: Proceeding of the NATO Advanced Research Workshop on Pulsed Metal Vapour Lasers - Physics and Emerging Applications in Industry, Medical and Science, St. Andrews (UK), Aug. 6-10, 1995 /

NATO ASI Series, Eds. Little C.E., Sabotinov N.V. Dordrecht, (1996).

[13] Buchkremer F .B. J., Andrews A. J., Coutts D. W., Webb C. E., "A new method for high speed imaging of particles within intensely radiating plasmas", Technical Digest of Papers Presented at The Thirteenth UK National Quantum Electronics Conference, University of Wales, Cardiff, 8-11 September, 116 (1997).

[14] Batenin V.M., Klimovskii I.I., Selezneva L.A., "Research of surfaces of electrodes of a carbon arc during its burning", Doklady Akademii Nauk (Proceedings of the Russian Academy of Sciences), 303 (4), 857-860 (1988).

[15] Abrosimov G.V., Polskiy V.V., Saenko V.B., "Using of a laser medium for photographing the surface hidden by layer of plasma" // Soviet Journal of Quantum Electronics, 15 (4), 850-852 (1988).

[16] Buzhinskij O.I., Vasiliev N.N., Moshkunov A.I., Slivitskaya I.A., Slivitsky A.A., "Copper vapor laser application for surface monitoring of divertor and first wall in ITER", Fusion Engineering and Design, (60), 141-155 (2002).

[17] Buzhinsky R.O., Savransky V.V., Zemskov K.I., Isaev A.A., Buzhinsky O.I., "Observation of objects under intense plasma background illumination”, Plasma Physics Reports, 36 (13), 1269-1271 2010.

[18] Abramov D. V. , Arakelyan S. M. , Galkin A. F. , Kvacheva L. D. , Klimovskii I. I. , Kononov M. A. , Mikhalitsyn L. A. , Kucherik A. O. , Prokoshev V. G., Savranskii V. V. , "Melting of carbon heated by focused laser radiation in air at atmospheric pressure and temperature below 4000 K”, Pis'ma v Zhurnal Ėksperimentalnoi I Teoreticheskoi Fiziki, 84 ( 5), 315-319 (2006).

[19] Arakelyan S.M., Prokoshev V.G., Abramov D.V., Kucherik A.O., [The microstructures, nanostructures and hydrodynamic instabilities induced by laser radiation on the surface of solids] [in Russian], Vladimir:Vladimir State University (2010).

[20] Yermachenko V.M., Kuznetsov A.P., Petrovskiy V.N., Prokopova N.M., Streltsov A.P., Uspenskiy S.A., "Specific features of the welding of metals by radiation of high-power fiber laser", Laser physics, 21 (8), 1530-1537 ( 2011). [21] Kuznetsov A.P., Gubskii K.L., Savjolov A.S., Sarantsev S.A., Terekhin A.N., Buzhinskij R.O., "Visualization of plasma-induced processes by a projection system with a Cu-laser-based brightness amplifier", Plasma Physics Reports, 36 (5), 428-437 (2010).

[22] Bokhan P.A., Dubnishcheva T.Ya., Zakrevskii D.E., Nastansha Yu.V., "Micron-image transfer system with brightness amplifier in gold-vapor laser", Journal of Russian Laser Research, 16, 164-171 (1995).

[23] Zemskov K.I., Kazaryan M.A., Petrash G.G., "Gold vapor brightness amplifier”, Optics Communications, 33 (2), 209-212 (1980).

[24] Arapova E. Ya. , Isaev A. A. , Kazaryan M. A., Markova S. V. , Petrash G. G. , Timofeev Yu. P. , Fridman S. A. , "Infrared laser projection microscope", Soviet Journal of Quantum Electronics, 2 (7), 1568-1570 (1975).

[25] Zinchenko S. P., Petrash G. G. , Sem M. F. , "Active optical systems with a helium-strontium recombination amplifier", Quantum Electronics, 20 (7), 677-679 (1993).

[26] Vlasov D.V., Ivashkin P.I., Isaev A.A., Kazaryan M.A., Kuznetsova T.I., and Chvykov V.V. "Amplification of Image Brightness in Strontium Vapor", Physica Scripta, 48, 461-463 (1993).

[27] Evtushenko G.S., Gubarev F.A., Sukhanov V.B., Shiyanov D.V., Torgaev S.N., Trigub M.V. "High-speed imaging of micro objects using the active media of metal vapor lasers under strong background radiation", Bulletin of Tomsk Polytechnic University, 315 (4), 141-146 (2009). 
[28] Evtushenko G. S., Trigub M. V., Gubarev F. A. , Evtushenko T. G., Torgaev S. N., Shiyanov D. V. "Laser monitor for non-destructive testing of materials and processes shielded by intensive background lighting", Review of Scientific Instruments, 85 (3), 1-5. (2014).

[29] Yusupov R.A., Kitler V.D., , Kirdyashkin A.I., Trigub M.V., Evtushenko G.S. "Dynamic monitoring of heterogeneous systems using laser radiation", Russian Physics Journal, 56 (9/3), 226-228 (2013).

[30] Rybka D.V., Trigub M.V., Sorokin D.A., Evtushenko G.S., and Tarasenko V.F. "Corona Discharge in Atmospheric Pressure Air when Using Modulated Voltage Pulses”, Atmospheric and Oceanic Optics, 27 (6), 582-586 (2014).

[31] Trigub M.V., Platonov V.V., Fedorov K.V., Evtushenko G.S., Osipov V.V. "CuBr-active medium for diagnostics of nano-powder producing process", XVI Conference on Diagnostics of High Temperature Plasma, Moscow-Zvenigorod, 7-11 June, 2015 and «Russian Physics Journal» (2015), (in press).

[32] Soldatov A. N. , Yudin N. A., Vasilieva A. V. , Kolmakov E. A. , Polunin Yu. P. , Kostyrya I. D. , "Strontium vapour laser with a pulse repetition rate of up to $1 \mathrm{MHz}$ ", Quantum Electronics, 42 (1), 31-33 (2012).

[33] Nekhoroshev V. O., Fedorov V.F., Evtushenko G.S., Torgaev S.N. "Copper bromide vapour laser with a pulse repetition rate up to $700 \mathrm{kHz}$ ", Quantum Electronics, 42 (10), 877-879 (2012).

[34] Boichenko A. M. , Evtushenko G. S. , Nekhoroshev V. O. , Shiyanov D. V. , Torgaev S. N. "CuBr-Ne-HBr laser with a high repetition frequency of the lasing pulses at a reduced energy deposition in the discharge", Physics of Wave Phenomena, 23 (1), 1-13 (2015)

[35] Trigub M. V., Shiyanov D. V., Sukhanov V. B. , Evtushenko G. S. "MnBr vapor active medium with a building reactor at $100 \mathrm{kHz}$ pulse repetition frequency", Atmospheric and Oceanic Optics, 27 (5), 458-462 (2014). 